\title{
Theoretical Interpretation of Solar and Stellar Irradiance Variations
}

\author{
By HENK C. SPRUIT
}

Max-Planck-Institut für Astrophysik, Postfach 1523, D-85740 Garching bei München, Germany

The main cause of variability of solar type stars are their varying magnetic fields. To compute irradiance variations one has to compute the magnetic field (the dynamo problem), and from this the irradiance effects. The second problem is considered here. The theoretical work of the past decade has shown that the dominant effect of magnetic fields is a surface effect: a change of effective emissivity of the magnetic parts of the surface while the nonmagnetic part of the surface contributes very little to the irradiance variation on almost all time scales. No other processes have yet been found that would cause variations exceeding (at the current level of magnetic activity) the observed $0.1 \%$ irradiance fluctuation of the Sun. This implies that a knowledge of the surface magnetic fields [separated into its bright small scale (faculae, network) and dark large scale (spots) components] is sufficient for pre- or postdicting the solar irradiance. It is hypothesized that the discrepancy remaining between the measured irradiance variations and values reconstructed from proxies is due to the difficulty of finding a proxy that accurately correlates with the continuum contrast of a dispersed small scale magnetic field. Stellar structure theory predicts that the variations in the solar radius associated with magnetic activity are quite small. For stars, color and brightness variations should primarily be interpreted in terms of variations in the fraction of the surface covered by magnetic patches. Their (long term) displacement from the main sequence is not very large.

\section{Introduction}

The solar luminosity varies on a wide range of time scales due to several processes. It increases as Hydrogen burns into Helium in the core. This increase is climatologically relevant $\left(\sim 30 \%\right.$ over the past $4.610^{9} \mathrm{yr}$ ), but does not cause changes on human time scales (see review by Kasting \& Grinspoon 1991). The Sun oscillates in a large number of sound modes, but their combined effect on irradiance is small $\left(\sim 10^{-5}\right.$ level, Fröhlich 1990; Fröhlich et al 1991) and confined to short time scales (minutes). The random pattern of the granulation at the surface causes statistical noise at an amplitude of a few times $10^{-5}$ and similar time scales. Larger scale convective flows are known to exist and have longer time scales, but their effect on irradiance probably does not exceed that of the granulation. This leaves magnetically related variations as the main known cause of variability on all time scales from hours to millions of years.

\subsection{Luminosity modulation by magnetic fields}

To make a successful theory of luminosity variations by magnetic fields one needs two ingredients. A theory is needed that tells us how much magnetic field is present and where, and given the distribution of magnetic fields, a theory to compute the resulting luminosity variations, during the Sun's magnetic cycle. The first of these questions is the dynamo problem. Dynamo theories have a long tradition and very extensive literature, but unfortunately have not progressed yet to the stage that the location and strength of the magnetic field in a star can be predicted. Hence one is forced to rely mostly on observations instead of theory. The second question turns out to be very much simpler. Once the magnetic fields are known, the problem of luminosity variations reduces to a calculation of thermal perturbations in which the sources are known. The theory of stellar structure is sufficiently well developed and tractable to produce reliable answers for this 
problem. These answers, and the underlying physics are the subject of the following sections.

Conceptually, it is easiest to separate the thermal effects of magnetic fields into two classes:

1. 'Sources and sinks'. The generation of a magnetic field involves the conversion of kinetic energy of motion into magnetic energy. Since the motions in the solar envelope are thermally driven, this ultimately means conversion of thermal into magnetic energy: building up a magnetic field produces a thermal sink somewhere. The opposite happens when the field decays. These thermal effects exist only during changes in the magnetic energy content of the envelope.

2. 'Changes in thermal transport coefficients'. Magnetic fields interfere with convection, causing a reduction in the efficiency of heat transport in the envelope. In contrast to (1), these changes last as long as the magnetic field itself is present.

Both these sources and sinks and changes in the transport coefficient cause thermal perturbations, varying with magnetic activity, which propagate through the envelope and cause variations in surface energy flux. Related to the second class of perturbations are the effects of magnetic fields at the surface of the star. Starspots, being dark, radiate less than the surrounding photosphere, while the small elements that make up plage and the network have an excess emission.

In the following I will refer mainly to the Sun, questions specifically relating to stars are discussed in Section 5.

\section{Time scales}

The response of the Sun to thermal perturbations is not governed by a single time scale, but by a wide range of time scales. The longest of these is the thermal time scale of the Sun as a whole, called the Kelvin-Helmholtz time scale, about $10^{7}$ years. If the central heat source of the Sun were switched off, the internal structure and the luminosity would start to change on this time scale. More generally, we can define the thermal time $\tau_{t}$ scale as a function of depth:

$$
\tau_{t} \equiv U(z) / L(z) \approx \frac{1}{L} \int_{R-z}^{R} 4 \pi r^{2} u \mathrm{~d} r,
$$

where $L$ is the luminosity at depth $z, U$ the thermal energy of the envelope down to a depth $z$, and $u$ the thermal energy per unit volume, approximately (ideal gas of constant $\gamma$ ) given by $u=P /(\gamma-1)$. Some rough values for this quantity are $\tau_{t} \sim 10^{5} \mathrm{yr}$ at $z=$ $210^{5} \mathrm{~km}$ (depth of the convection zone), $10 \mathrm{yr}$ at $20000 \mathrm{~km}$ (the size of a supergranule), $20 \mathrm{hrs}$ at $2000 \mathrm{~km}$ (size of a granule). This shows that the thermal time scale is a very sensitive function of depth in the Sun. As a result, the thermal response of the Sun also depends critically on the location of the disturbance. A fairly good approximation to the stratification of the convective envelope of the Sun is a polytrope of index $n=2$ (this is better than the standard 'convective' value $n=1.5$ because $\gamma<5 / 3$ due to partial ionization). Hence the gas pressure and thermal time scale vary roughly as $P \sim z^{3}$, $\tau_{t} \sim z^{4}$, where the depth $z$ is counted from a level 3 scale heights above the surface $\tau=1$.

A second kind of time scale involved in thermal readjustments is the diffusive time scale. In the mixing length approximation transport in the convection zone can be computed with a turbulent diffision coefficient $\kappa_{t} \approx \frac{1}{3} l_{c} v_{c}$ where $l_{c}$ and $v_{c}$ are the convective length scale and velocity. The time scale on which thermal inhomogeneities (more precisely: 
entropy perturbations) are smoothed on a length scale $d$ by turbulent diffusion is

$$
\tau_{d}=d^{2} / \kappa_{t} \text {. }
$$

For $d=210^{5} \mathrm{~km}$ this is about $1 \mathrm{yr}$, for $d=2000 \mathrm{~km}$ about $1 \mathrm{hr}$. Comparing $\tau_{t}$ and $\tau_{d}$, we see that they are of similar magnitude close to the surface (to be precise: in the surface boundary layer where convection is not efficient enough to keep the stratification close to adiabatic). In deeper layers, the thermal time scale is much longer than the diffusive time scale, by a factor of up to $10^{5}$. The two time scales measure different types of thermal adjustment process. These same processes appear in the thermal behavior of, say, a spacecraft. Let me approximate a satellite as a chunk of aluminum with a heater in it. The thermal time scale is the time scale on which its temperature adjusts to a change in the heat input, and is determined by the heat capacity [ $U$ in eq. (2.1)] and the power level $(L)$. The time scale on which different parts of the chunk equilibrate to the same temperature is governed by a different process, namely the thermal conduction (the equivalent of the turbulent diffusion in the Sun). The diffusion (conduction) time scale is much shorter than the thermal time scale, because of the large conductivity of Al. In the Sun, it is the very large turbulent diffusivity in the bulk of the convection zone that causes the very short diffusive time scale compared with the thermal time scale.

How do these different time scales come into play when the convection zone is thermally perturbed by, say, the storage of energy in a growing magnetic field? Such perturbations can be computed in detail, either by numerical methods (Endal et al. 1985) or more analytically. We can, for example, consider the initial value problem in which a perturbation is allowed to evolve in time by thermal transport in the convection zone. In general this evolution has components on all the time scales of the problem, including the very long thermal time scale. Formal aspects of this problem are discussed briefly in the next subsection (for a more complete analysis, see Spruit 1982a,b, 1991; Arendt 1992). For quantitative results, skip to Section (2.2).

\subsection{Modes of thermal relaxation}

The problem can be studied in detail within the mixing length approximation for convective energy transport:

$$
\mathbf{F}=-\kappa \rho T \nabla S
$$

where $\mathbf{F}$ is the heat flux, $\kappa$ the turbulent diffusivity, $\rho$ the density, $T$ the temperature and $S$ the entropy. The energy equation, in the absence of sources, is given in terms of the entropy by

$$
\rho T \frac{\mathrm{d} S}{\mathrm{~d} t}=-\operatorname{div} \mathbf{F}
$$

As an example, consider the one dimensional problem in which all quantities depend on depth $z$ only. In this case, combining (2.3) and (2.4), and neglecting flows we get

$$
\frac{\partial S}{\partial t}=\frac{F_{z}}{\rho T H}+\kappa \frac{\partial S}{\partial z^{2}},
$$

where $H=\partial \ln \rho T / \partial z^{-1}$ is the pressure scale height. For perturbations in which the left hand side is balanced by the first term on the right, the time scale is of the order $F /\left(c_{p} \rho T H\right)$, which is just a thermal time scale (cf. 2.1). For modes in which the left hand side is balanced by the second term, we evidently have an ordinary diffusion problem, hence these modes evolve on diffusive time scales (cf. 2.2). The first term appeared as a consequence of the stratification of pressure, ie. the inhomogeneity of the convection zone. In a homogeneous medium (as in a chunk of $\mathrm{Al}$ ), the thermal time scale comes in 
only through the radiating surface boundary condition (which one could also consider as a form of inhomogeneity). If $T_{s}$ is the surface temperature, this condition is

$$
\sigma T_{s}^{4}=F_{z} .
$$

Since the perturbations in entropy outside spots and the magnetic elements making up the small scale field are small, the reponse of the convection zone can be computed by linearizing eqs. (2.3),(2.4),(2.6). An arbitrary initial perturbation may then be decomposed in the standard way in terms of the eigenmodes of the problem. These are found by setting $\delta S=e^{\eta t} f(\mathbf{r})$, and solving for $f(\mathbf{r})$ with the decay rate $\eta$ as eigenvalue (the problem thus defined has only decaying solutions). Specializing to the one dimensional case $(f(z))$ one finds a series of modes ordered by the number of nodes $n$ of $f$. The fundamental $(n=0)$ has a decay rate of the order $\eta_{0} \sim \tau_{t}^{-1}$ that is, this mode decays on the thermal time scale. The higher modes decay with rates of the order $\eta_{n} \sim n^{2} \tau_{d}^{-1}$, they decay on diffusive time scales.

\section{Effect of subsurface magnetic fields on luminosity}

In this section I consider the effect on luminosity of magnetic fields in the convection zone which vary on the time scale $t_{c} \approx 10 \mathrm{yr}$ of the solar cycle (for a discussion of more general perturbations and for details of the calculations, see Spruit 1982a,b 1991). The best current guess is that most of the flux, of the order $10^{23}-10^{24}$ (Zwaan 1978) $\mathrm{Gcm}^{2}$ is located near the base of the convection zone, and has a strength of the order $50-100 \mathrm{kG}$ (D'Silva \& Choudhuri 1993; D'Silva \& Howard 1993). These quantities are somewhat model dependent.

To reduce the dependence on these models, let me keep the depth $z_{b}$ at which the field is located as a free parameter. To obtain upper limits to the luminosity effects, assume that the field at $z_{b}$ covers the entire horizontal surface, and covers a depth range of one pressure scale height $H$ (this is sufficiently general for the present purpose, since $H\left(z_{b}\right)$ is a significant fraction, of the order $1 / 3$, of $z_{b}$ itself). The convection zone, being nearly isentropically stratified, offers no restoring force to the motion of the magnetic field. If the field is in temperature equilibrium with its surroundings, the gas density in it is less, causing magnetic buoyancy. The speed at which the field starts rising under the influence of this force is of the order of the Alfvén speed. If the field is to remain inside the convection zone for a significant fraction of the cycle time, this yields the so-called buoyancy limit on the field of the order of $100 \mathrm{G}$ (Parker 1984). Buoyancy can be avoided by creating the field with a slight temperature deficit, in such a way that it is neutrally buoyant. Such fields are found to be unstable however, on an Alfvén travel time scale (Parker 1979, 1984; Spruit \& van Ballegooijen 1982). This again limits the field to the buoyancy limit. This restrictive limit can be relaxed a bit by noting that convective downflows exist that are larger than the Alfven speed at 100G. In principle, these flows can be used to prevent a field from rising through the convection zone. Making this assumption, the upper limit to the field becomes equal to the equipartition value, at which the energy density in the field equals the kinetic energy of the convective flows. This value increases with depth, to a maximum of about $10000 \mathrm{G}$ near the base of the convection zone.

With these values for the strength, extent and location of the field, the predicted upper limits to fluctuations in luminosity can be estimated using the formalism of Section (2.1). This is shown in Figure 1. The results depend on the nature of the perturbations. The result of conversion between thermal and magnetic energy is called a ' $\beta$-perturbation', that caused by a reduction of the convective energy transport efficiency an ' $\alpha$-perturbation' 

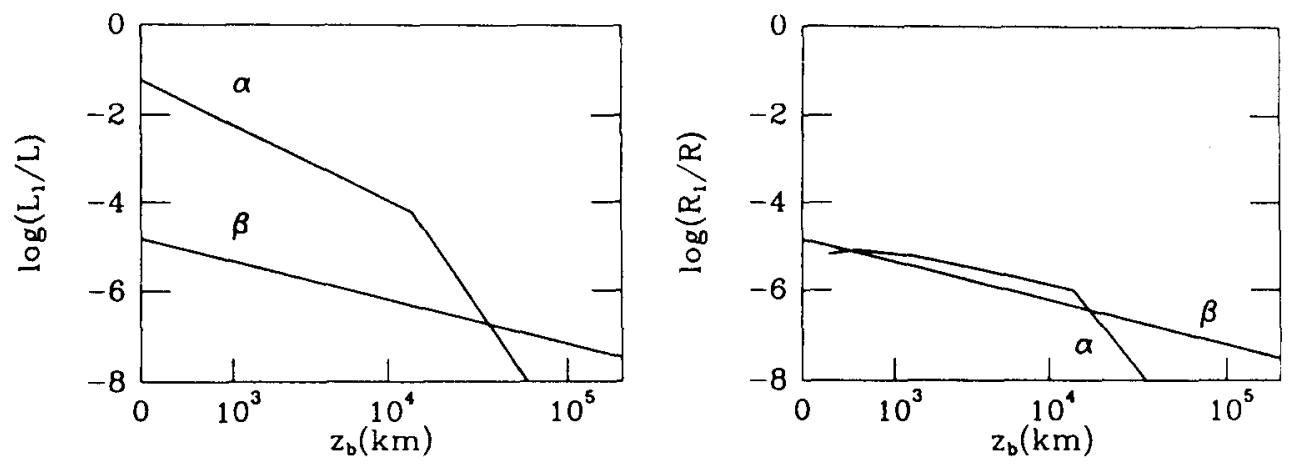

FIGURE 1. Upper limit on luminosity and radius variations on a solar cycle time scale caused by magnetic fields in the convection zone, as a function of their assumed depth $z$, for fields limited by equipartition with convection

(Endal et al. 1982). In computing the $\alpha$-perturbations, it is assumed that the reduction of convective efficiency by the field is of the order unity if the field strength is equal to the equipartition value (Spiegel \& Weiss 1980). From Figure 1 it is clear that $\alpha$-perturbations are the more effective ones (Gilliland 1988) except near the base of the convection zone. This has to do with the fact that the field can interfere with the convective flow as long as it exists, whereas the $\beta$-effect is limited by the magnetic energy content of the field. [Near the base the relative effectiveness of the two is reversed because convection there is so efficient that a reduction by the field has little effect]. The results in Figure 1 agree qualitatively with results obtained from perturbed stellar structure models (e.g. Endal et al. 1982; Gilliland 1988).

Figure 1 shows that luminosity variations on the order of the observed $0.1 \%$ irradiance variation can not be caused by subsurface magnetic fields unless they are located at a depth less than a few thousand $\mathrm{km}$. This is so close to the surface that observations probably give a better estimate of the magnetic field at this depth than the theoretical limits used above. The surface field is nearly vertical, $1-3 \mathrm{kG}$, and generally has a filling factor of the order $1 \%$ or less. Because of the low filling factor (compared with the $100 \%$ filling factor assumed so far), such fields have much smaller effect than the fields assumed above (see Section 4).

At the base of the convection zone, the upper limit on the field strength based on the buoyancy argument is not valid, since the radiative core is quite stably stratified, and acts as a nearly solid surface towards motions in the convection zone. Fields close to this surface could plausibly be higher than the equipartion value. Ignoring the buoyancy limit, the next most plausible upper limit to the field strength is obtained by assuming that the entire luminosity of the Sun is spent in building up a magnetic field near the base of the convection zone during the ascending phase of the solar cycle, and this same amount released again during the descending phase. One finds that the luminosity effect in this case is $\delta L / L<10^{-5}$ (for $\beta$-perturbations, which are the most effective ones in this case). This perhaps surprising result has to do with the very large heat capacity of the convection zone, related to its thermal time scale of $10^{5} \mathrm{yr}$.

The variations in solar radius predicted are small. The maximum values in Figure 1 may be observable, but if we take the current estimate (see above) that most of the field is located near the base of the convection zone, a plausible estimate is $\delta R / R \sim 10^{-7}$. The vertical magnetic fields resulting from eruption of active regions have an additional 
effect on $\delta R$, but this is even smaller. These radius variations are defined as changes of the nonmagnetic part of the solar surface. In spots and small magnetic elements, the $\tau=1$ surface is depressed (Wilson depression). If one were to compute a mean geometric radius including these depressions, radius changes up to $10^{-5}$ (a reduction at solar maximum) would result. This mean radius is not directly observably however, and the radius of the nonmagnetic photosphere is, also observationally, the more meaningful quantitity.

\section{Effect of surface magnetic fields}

The strongest effects on luminosity are obtained from fields at the radiating surface itself (cf. Figure 1). This is mostly a reflection of the steep depth dependence of the thermal inertia of the convection zone. The surface effects are caused by changes in the efficiency of energy transport associated with the field. A reduction of the convective energy flux due to the resistance of magnetic field lines against bending causes sunspots to have a (bolometric) surface emissivity of about $20-25 \%$ of that of the normal solar surface. In very small magnetic concentrations (less than about three pressure scale heights across) this is more than compensated by a net excess emissivity. Due to the lower internal pressure such concentrations are small 'pits' in the surface. Through the sides of these depressions, an extra energy flux is emitted, which is visible mainly when viewing them at an angle from the vertical i.e. when seen near the limb (Spruit 1976, 1977). Most of the magnetic field on the solar surface is in the form of these small concentrations, and it appears from the irradiance measurements of the last cycle that their effect dominates over the reduced emission of sunspots (this may not have been the case in all cycles, however, see Foukal \& Lean 1990). Theoretical prediction is difficult, since the excess emission from a facular area is a sensitive function of the poorly known size distribution of the concentrations (see however Spruit \& Zwaan 1981). For a given size, the effect can be computed in principle through detailed numerical simulation of the energy transport in the concentration and its surroundings. Such calculations have already been undertaken by Knölker et al. (1991); Schüssler (1991).

Changes in surface emissivity are much more effective than magnetic energy storage or release in the same structures. Consider for example a small concentration with a contrast $\phi$ (averaged over its surface cross section). The life time of such elements can be estimated from the decay time of an active region, on the order of a few months. During this time $\tau$, the element emits, per unit of magnetic flux, an excess energy $E_{e}=\phi \tau F_{\odot} / B_{s}$, where $B_{s}$ is the surface magnetic field strength. If the field lines of the element reach down to base $z=D$ of the convection zone, their magnetic energy content per unit magnetic flux is $E_{B}=D B / 8 \pi$. Putting in $B \sim 2 \mathrm{kG}$, one finds that $E_{e}$ is more than a factor 10 larger than $E_{B}$. This is only a simple energetic argument. In practice, the magnetic energy content of a magnetic structure is not so simply available to be emitted at the surface. In fact, there is no known way of transporting the energy in the deeper layer of the magnetic structure to the surface on the required time scale, even if all of it were available. This contrasts with the effect of the field on the surface emissivity. This effect develops as soon as the magnetic structure appears at the surface, because of the very short thermal time scale of the surface layers.

We conclude from the discussion so far that changes of the surface emissivity are predicted to be the main cause of irradiance variations. Since the brightness of sunspots and small scale fields can be measured, an irradiance variation can be calculated by just multiplying contrasts and areas and adding up. Is the situation as simple as this? What if some of the heat flux blocked by sunspots for example were reemitted elsewhere on the 
surface in diluted form? Calculations based on the formalism mentioned in Section (2.1) predict that, in fact, this reemission 'at a distance' does not take place (Foukal et al. 1983; Spruit 1982a,b; Chiang \& Foukal 1984), on time scales of human interest. On time scales of the order $\tau_{t} \sim 10^{5} \mathrm{yr}$, the blocked flux does reappear. It does so in distributed form, nearly uniformly over the surface. Thus, the current nonmagnetic surface of the Sun radiates the blocked flux of all the spots on its surface avaraged over the past $10^{5} \mathrm{yr}$ (and similarly for the small scale fields). Due to this averaging effect, the nonmagnetic surface does not change on observable time scales.

Since the contrasts and numbers of magnetic elements of various sizes at the surface are in principle measurable at any point in time, the prediction that the brightness nonmagnetic surface does not take part in the cycle can in principle be checked. In practice this is rather difficult, since the largest effect comes from the small scale field. The predicted net emission of these small scale structures is a very sensitive function of their size (Spruit 1977; Spruit \& Zwaaan 1981), and the largest effect is predicted from structures that are near or below the current spatial resolution. To account for the small scale field meaningfully, an intensive study of the effective brightness of the small scale field, and its magnetic flux has to be made, as a function of its evolutionary state (since it is known that the typical size of magnetic elements is larger in young active regions than in old ones, for example). The current proxy indicators do not represent the relevant quantity (photospheric brightness) accurately enough. The prediction that the smallest elements give the largest contribution would mean that the contribution of the most dispersed small scale fields, which are hard to observe, may have been strongly underestimated. The results of Lean et al. $(1992,1994)$ point in this direction.

\subsection{The 'blocked heat flux problem' of sunspots}

Suppose at $t=0$ a spot appears instantly on the surface, reducing the heat flux. At least a part of the blocked heat flux will find its way 'around' this obstacle, to show up as a 'bright ring'. How long does it take for this to happen, and what fraction of the blocked heat flux is reemitted elsewhere? To see this, consider a terrestrial analogy, consisting of an aluminum brick heated from below, and losing heat to its environments from the top. On this I put, again at $t=0$, a thermally insulating patch. Does a 'hot ring' develop around the patch? Is all of the heat flux blocked by the patch radiated from some other part of the surface? The answer is no. The high thermal conductivity of aluminum does not allow strong inhomogeneities like a hot ring. By conduction an even temperature is maintained, and the blocked heat flux, instead of reappearing somehwere on the surface, appears in the energy balance as a slow heating up of the brick. A new equilibrium is reached on the thermal time scale of the brick, which is long on account of its high heat capacity. This situation also applies to a convective stellar envelope, with only a small additional complication. In a convective envelope both the thermal (turbulent) conductivity and the heat capacity are strong functions of depth. The comparison with aluminum applies only at depths where the convective stratification is close to adiabatic. Above this, convection is less efficient, and the heat capacity small. For this reason, it matters how deep into the envelope the blocking object extends. For the Sun, one finds (Spruit 1982a; Foukal et al. 1983) that less than $5 \%$ of the blocked flux reappears at the surface, on time scales of observational interest $\left(<10^{5} \mathrm{yr}\right)$, if the blocking extends to a depth greater than about $1000 \mathrm{~km}$. The part that does return to the surface does so very close to the spot or facular element.

The prediction is thus that in practice the solar surface outside magnetic regions does not take part in irradiance variations associated with the solar cycle. For dissenting 
views on the spot blocking problem and related matters, see Chapman et al. (1984), Sofia et al. (1982), Schatten \& Mayr (1985), Fox et al. (1991), and Kuhn (1994).

In numerical simulations of spot blocking (Foukal et al. 1983; Fox et al. 1991), the very large range of time scales causes problems. This is one of the reasons why the depth of the convection zone is reduced artificially in such calculations. As a result, the thermal time scale in such calculations is also much shorter than in the real convection zone. In the simulation by Fox et al. (1991) with a depth of only $2000 \mathrm{~km}$ the thermal time scale is on the order of a few hours instead of $10^{5} \mathrm{yr}$ for the real convection zone. The rapid return to a steady heat flux observed by Fox et al. is a consequence of this shallow depth. Secondly, the depth of their 'spot', $150 \mathrm{~km}$, is much shallower than a real spot. The Wilson depression alone of a spot is about four times as deep as this. The strength of the sunspot field, $3000 \mathrm{G}$, is large enough to interfere strongly with the convective heat flow for several thousand $\mathrm{km}$ below the surface. Whereas a convective diffusion model predicts that less than $10 \%$ of the blocked heat flux returns to the surface if the blocking effect extends $1000 \mathrm{~km}$ or more (Spruit 1982a), it predicts that $60 \%$ returns if the blocking is only $150 \mathrm{~km}$ deep. This is the consequence of the very steep increase of the turbulent thermal conductivity with depth (roughly proportional to $\rho$ ). Finally the upper boundary condition used by Fox $e t$ al., which sets the temperature fluctuations to zero at the top surface instead of a proper radiative boundary condition, overestimates the fraction of the blocked heat flux that returns to the surface (Spruit 1976, Section 4.2). In other words the results by Fox et al. are in qualitative agreement with the diffusion model, and do not say much about sunspots.

For small magnetic concentrations, in which a lateral influx of heat enters into the tube rather close below the solar surface, a significant fraction of the influx of heat may derive from the immediate surroundings (forming a 'dark ring') around it. The extent to which this happens depends on the precise nature of the energy carrying flow near the tube, which is the subject of current numerical study. Since the concentrations that show a net excess are so small, the measured excess is usually an average over the concentration (see however Keller 1992). This average can still be positive, because part of the lateral influx derives from the interior of the convection zone (this is a consequence of the rapid increase of the thermal conductivity with depth). In a simple diffusion model for the heat transport near the tube, an average excess emissivity of up to $100 \%$ was found (Spruit 1977, Figures 16,17, see also Spruit \& Zwaan 1981).

\section{Stars}

Since main sequence stars of type $\mathrm{F}$ and later are known to have spots and chromosheric emission like the Sun (for reviews see, e.g. Byrne \& Mullan 1992), we may endeavor to extrapolate the conclusions in the preceding sections to these stars. One predicts then that the nonmagnetic part of the stellar surface does not change in brightness or color on observable time scales, except perhaps in early F-stars in which the convection zone is much shallower than in the Sun. Variations in brightness and colors of these stars, whether due to rotational modulation or to changing magnetic fields, can then be interpreted directly in terms of the color properties of the magnetic structures involved and their distribution on the surface. Even secular changes in brightness on time scales of centuries do not involve the nonmagnetic surface. Since the umbrae of spots are rather dark in the visual range, they contribute relatively little to color changes; more effective are the penumbrae (cf. Spruit \& Weiss 1986). This depends on the size of starspot penumbrae however, about which little is known.

For the position of an active star in the Hertzsprung-Russel Diagram (HRD), changes 
in the nonmagnetic part of the surface do play a role, since the age of the star is much longer than the thermal time scale of the convection zone. The brightness of the nonmagnetic surface in these stars reflects the average of the magnetic activity over the last thermal time scale of its convection zone. Changes in position in the HRD, taking this into account, have been computed by Spruit \& Weiss (1986), assuming spots with properties similar to sunspots. The stars were found to remain close to the main sequence, even for spots covering a significant farction of the stellar surface, because the displacement in the HRD is nearly parallel to the main sequence.

\section{Conclusions}

The theory of structure and evolution of stellar envelopes gives a rather simple picture for the effect of magnetic fields on luminosity (irradiance). This is because it predicts that for irradiance variations on time scales short compared with $10^{5} \mathrm{yr}$ the non-magnetic surface participates only at a very low level in the variations due to magnetic fields. Taking account of effects that are ignored in the calculations, such as systematic flows and uncertainties in the mixing length model used, may certainly change the contribution by factors of order unity. The main conclusion is robust with respect to such changes because this contribution is so small in the first place. Most of the uncertainty in making comparisons with observations comes from our poor knowledge of the intrinsic brightness and magnetic flux in the small scale magnetic field, especially in its most dispersed form, since this field contributes disproportionately to the net irradiance. Its brightness for a given size and shape of the magnetic elements can in principle be obtained from numerical simulations (Knölker et al. 1991). The spectrum of scales in the small scale field however depends on aspects of the solar dynamo process that are intrinsically difficult and have hardly been adressed theoretically.

Acknowledgments. I thank Dr. Sami Solanki and an anonymous referee for corrections and useful comments.

\section{REFERENCES}

ARENDT, S. 1992 Large-scale flows and solar luminosity variations. Astrophys. $J$ 389, 421-427.

BYrne, P. B. \& Mullan, D. J. 1992 Influence of starspots on internal stellar structure. In Lecture Notes in Physics 397, 78-84. Springer-Verlag.

Chapman, G. A., Herzog, A. D., Lawrence, J. K. \& Shelton, J. C. 1984 Solar luminosity fluctuations and active region photometry. Astrophys. J. 282, L99-L101.

Chiang, W. H. \& FoukaL, P. V. 1984 The influence of facuale on sunspot heat blocking. Solar Phys. 97, 9-20.

D'Silva, S. \& Choudhuri, A. R. 1993 A theoretical model for tilts of bipolar magnetic regions. Astron. Astrophys. 272, 621-633.

D'Silva \& Howard, R. A. 1993 Limits on the magnetic field strength at the base of the solar convective zone. Solar Phys. 148, 1-9.

bitem[]Endal, A.S., Sofia, S. \& Twigg, L.W. 1982 Changes of solar luminosity and radius following secular perturbations in the convective envelope. Astrophys. J. 290, 748757.

FouKAL, P. V., \& LEAN, J. 1990 An empirical model of total solar irradiance variations between 1874 and 1988. Science 247, 556-558.

$\dagger$ To be precise: the surface outside about 1 granule diameter from a magnetic element, since the magnetic field has some influence on the convective flow in its neighborhood. 
Foukal, P., Fowler, L.A. \& Livshits, M. 1983 A thermal model of sunspot influence on solar luminosity. Astrophys. J. 267, 863-871.

Fox, P., Sofia, S., Chan, K. L. 1991 Convective flows around sunspot-like objects. Solar Phys. 135, 15-42.

FröHLICH, C. 1990 Irradiance variability of the Sun. In Climate Impact of Solar Variability (ed. K. H. Schatten \& A. Arking) NASA CP 3086, pp. 269-278.

Fröhlich, C., Foukal, P. V., Hickey, J. R. \& Hudson, H. S., Willson, R. C. 1991 Solar irradiance variability from modern measurements. In The Sun in Time (ed. C. Sonett, M. Giampapa \& M. Matthews). pp. 11-29. University of Arizona, Tucson, AZ, USA.

Gilliland, R. L. 1988 In Solar radiative output variation, Cambridge Research \& Instrumentation Inc., P. Foukal, ed., 21 Erie st. Cambridge, MA 02139, pp. 239.

Kasting, J.F., \& Grinspoon, D.H. 1991 The faint young Sun problem. In The Sun in Time (ed. C. Sonnett, M. Giampapa \& M. Matthews). University of Arizona, Tucson, pp. 447462.

KeLler, C.U. 1992 Resolution of magnetic flux tubes on the Sun Nature 359, 307-308.

Knölker, M. Grossmann-Doerth, U., Schüssler, M., WeisshaAr, E. 1991 Adv. Sp. Res. $11,285$.

KunN, J. 1994 Brightness observations of the Sun. In The Sun as a Variable Star: Solar and Stellar Irradiance Variations (ed. J.M. Pap, C. Fröhlich, H.S. Hudson \& S.K. Solanki). Cambridge University Press, in press.

Lean, J., Skumanich, A. \& White, O.R. 1992 Estimating the Sun's radiative output during the Maunder Minimum. Geophys. Res. Lett. 19, 1595-1598.

Lean, J., SKumanich, A., White, O.R. \& Rind, D. 1994 Estimating solar forcing of climate change during the Maunder Minimum. In The Sun as a Variable Star: Solar and Stellar Irradiance Variations (ed. J.M. Pap, C. Fröhlich, H.S. Hudson \& S.K. Solanki). Cambridge University Press, in press.

Parker, E. N. 1979 Cosmical Magnetic Fields Oxford, Clarendon Press.

PARKer, E. N. 1984 Magnetic buoyancy and the escape of magnetic fields from stars. Astrophys. J. 281, 839-845.

Schatten, K. H. \& MaYR, H. G. 1985 On the maintenance of sunspots - An ion hurricane mechanism. Astrophys. J. 299, 1051-1062.

Schüssler, M. 1991 Geophys. Astrophys. Fluid Dyn. 62, 271.

Sofia, S. Schatten, K.H. \& Oster, L. 1982 Solar irradiance modulation by active regions during 1980. Solar Phys. 80, 87-98.

SPIEgEL, E.A. \& WEIss, N.O. 1980 Magnetic activity and variations in solar luminosity. Nature 287, 616-617.

SPRUIT, H.C. 1976 Pressure equilibrium and energy balance of small photospheric flux tubes. Solar Phys. 50, 269-295.

SPRUIT, H.C. 1977 Heat flow near obstacles in the solar convective zone. Solar Phys. 55, 3-34.

SPRUiT, H.C. 1982a Effect of spots on a star's radius and luminosity. Astron. Astrophys. 108, 348-355.

Spruit, H.C. $1982 \mathrm{~b}$ The flow of heat near a spot. Astron. Astrophys. 108, 356-360.

Spruit, H.C. 1991 In The Sun in Time (ed. C. Sonett, M. Giampapa \& M.S. Matthews). pp. 118-158. University of Arizona Press, Tucson, AZ, USA.

SPRUIT, H.C. \& ZWAAN, C. 1981 The size dependence of contrasts and numbers of small magnetic flux tubes in an active region. Solar Phys. 70, 207-228.

Spruit, H.C. \& van Ballegooijen, A.A. 1982 Stability of toroidal flux tubes in stars. Astron. Astrophys. 106, 58-66, Erratum in 113, 350.

Spruit, H.C. \& WeIss, A. 1986 Colors and luminosities of stars. Astron. Astrophys. 166, 167-176.

ZWAAN, C. 1978 On the appearance of magnetic flux in the solar photosphere. Solar Phys. 60, 213-240. 